\title{
Misrepresentation of Dutch neuter gender in older bilingual children?
}

Susanne Brouwer, Leonie Cornips and Aafke Hulk

Max Planck Institute for Psycholinguistics, Nijmegen /

Meertens Institute (KNAW), Amsterdam / University of Amsterdam

Previous research revealed that monolingual children between 11- and 13-yearold show a target-like production with respect to gender assignment of definite determiners whereas this is not the case for bilingual children who massively overgeneralize $d e$. In order to further investigate this overgeneralization, we designed an experimental decision task, "tapping the knowledge" of both monolingual and bilingual children. Our results show that bilinguals fail to represent abstract gender and that $d e$ and het are in free variation. The difference between the production and comprehension data could suggest that bilinguals have some awareness of $d e$ and het being gender markers. In that case, the overgeneralization in the production data is possibly not a grammatical phenomenon, but more likely a speech production strategy.

\section{Introduction}

Unlike English, Dutch draws a distinction between common and neuter nouns. Grammatical gender is reflected in a number of agreeing elements accompanying the noun or referring to it. Definite determiners are a clear case: singular definite determiners vary morphologically according to the gender of the noun, as illustrated in Table 1 below. Nouns that take the singular definite determiner de, such as de hond 'the dog' are called de-words and can be referred to as being common gender. Nouns that take the singular definite determiner het, such as het schaap 'the sheep', are called het-words, and can be referred to as having neuter gender. ${ }^{1}$

1. Similar to definite determiners, demonstrative determiners vary morphologically according to the gender of the accompanying noun in the singular. Further, attributive adjective agreement always requires a schwa $(+e)$, except with nouns that are [indefinite, singular, neuter]. 
Table 1. Dutch morphology of definite determiners

\begin{tabular}{lll}
\hline Gender of noun & \multicolumn{2}{c}{ Definite determiners } \\
& Singular & Plural \\
\hline Common & de & de \\
Neuter & het & de \\
\hline
\end{tabular}

Spontaneous as well as experimental production data regarding the acquisition of the gender of definite determiners in monolingual Dutch child language reveals that children until the age of six have serious problems with neuter gender (cf. Bol \& Kuiken 1988; De Houwer \& Gillis 1998). Furthermore, bilingual children who are simultaneously acquiring two languages from birth (Dutch and another one) have been shown to present even more problems with the acquisition of neuter gender, and during a longer time irrespective of their other language. In previous papers, we have pointed out that the Dutch two-gender system is not very salient for learners of Dutch because there is a lot of syncretism in the paradigms. This might be one of the reasons why het is problematic (Hulk \& Cornips 2006a, b; Cornips et al. 2006).

The question that arises is whether these problems are restricted to (a mapping problem involving) 'production' strategies or whether they reflect a lack of knowledge. The aim of this paper is to get more insight in this question. We designed an experimental decision task that should provide us with more information about knowledge of the gender of the definite determiner in Dutch. Our subjects are monolingual and bilingual children between 11 and 13 years old. We will consider two hypotheses that arise from the production data of bilingual children (Blom, Polisenská, \& Weerman 2006; Hulk \& Cornips 2006a, b). First, if bilingual children have a mapping problem, but not a representational problem then they will differ from monolinguals in speech, overgeneralising one of the article forms as a default. By contrast, in a decision task (which appears to involve comprehension rather than production) they should not differ from monolingual peers in their response patterns.

Second, if bilingual children have a representational problem and not a mapping problem, their responses should differ from monolinguals both in speech and in the decision task. Our research question is whether we find support for either one of these hypotheses.

The paper is organized as follows. In the next section, we briefly review relevant production data for monolingual and bilingual children from the literature. Based on the bilingual production results, two explanations are put forward. These hypotheses attempt to find an answer to the question of what knowledge bilingual children possess about the gender of the Dutch definite determiner. In
Section 3, a new experimental design, eliciting both accuracy and response time data, is proposed to "tap the knowledge" of bilingual subjects in the age range between 11 and 13 years old. Section 4 presents the accuracy and response time data for the two language groups. Finally, Section 5 summarizes the main points and gives new interpretations regarding the gender acquisition of definite determiners in bilingual children.

\section{Production data}

Production data have revealed that monolingual acquisition of neuter gender of the Dutch definite determiner is a long-lasting process in the sense that children do not acquire a target grammar until a very advanced age (cf. Bol \& Kuiken 1988; De Houwer \& Gillis 1998), to be more specific, not before the age of six (cf. Van der Velde 2002, 2004). Interestingly, monolingual Dutch children clearly differ from their international peers since they overgeneralize in one direction only and adopt $d e$ as compatible with both neuter and common nouns. Thus, monolinguals overgeneralize the definite determiner $d e$, as required by common nouns in adult grammar (see Table 1 in Section 1), and use it incorrectly with neuter nouns that require the definite determiner het.

It is expected that when monolingual children have problems acquiring a certain phenomenon, we can be sure that bilingual children encounter even larger problems. Indeed, results of a picture description task by Blom et al. (2006) (see also Hulk \& Cornips 2006a, b for younger bilingual i.e. Turkish and Moroccan children and Cornips et al. 2006) for older Turkish and Moroccan children demonstrate that both age groups show severe difficulties regarding the acquisition of neuter gender in their elicited production of the definite determiner, as illustrated in Tables 2 and 3 respectively.

First, the monolingual children between 3 and 5 years old show overgeneralization of $d e$ in one direction ( $d e$ is used with het-nouns in $52 \%$ of the cases). The older monolingual children between 11 and 13 years, however, produce de almost target-like. Second, the youngest bilingual children also use de as compatible with both neuter $(60 \%)$ and common nouns $(63,5 \%)$ (see Table 2). The older bilingual children differ from the older monolingual children in that the former still overgeneralize $d e$ with neuter nouns although to a slightly lesser extent $(48,9 \%){ }^{2}$

2. We will discuss the production of het below. 
Table 2. Monolingual and bilingual results for the determiner de/het, age between 3-5 years (target in bold); taken from Blom, Polisenská \& Weerman (2006)

\begin{tabular}{|c|c|c|c|c|}
\hline & \multicolumn{2}{|c|}{ Monolinguals $(\mathrm{N}=39 ; 3-5 \mathrm{yr})$} & \multicolumn{2}{|c|}{ Bilinguals $(\mathrm{N}=30 ; 5-8 \mathrm{yr})$} \\
\hline & de & het & de & het \\
\hline Neuter & $52 \%$ & $44 \%$ & $60 \%$ & $4 \%$ \\
\hline Common & $88.5 \%$ & $6.5 \%$ & $63.5 \%$ & $3.5 \%$ \\
\hline
\end{tabular}

Table 3. Monolingual and bilingual results for the determiner de/het, age between 10;5 and 12;11 years (target in bold); taken from Cornips et al. (2006)

\begin{tabular}{lllll}
\hline & \multicolumn{2}{l}{$\begin{array}{l}\text { Monolinguals }(\mathrm{N}=4 ; 10 ; 5-12 ; 11 \\
\text { de }\end{array}$} & het & \multicolumn{2}{l}{$\begin{array}{l}\text { Bilinguals }(\mathrm{N}=24 ; 10 ; 5-12 ; 11 \\
\text { }\end{array}$} & de $)$ & het \\
\hline Neuter & $18.7 \%$ & $68.8 \%$ & $48.9 \%$ & $42 \%$ \\
& $9 / 48$ & $33 / 48$ & $141 / 288$ & $121 / 288$ \\
Common & $83.3 \%$ & $4.2 \%$ & $68.7 \%$ & $23.6 \%$ \\
& $40 / 48$ & $2 / 48$ & $198 / 288$ & $68 / 288$ \\
\hline
\end{tabular}

Taken together, in contrast to the monolingual children, the bilingual children still largely overgeneralize $d e$ when they are between 10 and 13 years old. ${ }^{3}$

\section{Present study}

3.1 Subjects

In this experiment a total of 42 children in the age range between 11 and 13 years old participated. There were two language groups: monolingual $(n=18)$ and bilingual $(n=24)$ children. ${ }^{4}$ The subjects were recruited from four different primary schools in the east of the Netherlands. The bilingual subjects all belong to the same group level common in the school system and were selected by the teachers on the basis of their proficiency in Dutch.

3. In this article we will leave aside the question which theoretical explanation can be offered for the overgeneralization of $d e$, but see the conclusion section for some speculative suggestions.

4. In this study we did not consider the possible influence of the other languages of the biThere we found no influence of the other language (Hulk \& Cornips 2006a, b; Cornips et al. 2006)
Table 4. Overview of experimental items

\begin{tabular}{lll}
\hline Gender of noun & \multicolumn{1}{c}{ Presentation } & \\
& Correct & Incorrect. \\
\hline Common & $\begin{array}{l}\text { Ik zie de hond (12) } \\
\text { 'I see the dog'. CorrectDE }\end{array}$ & 'Ik zie het hond (12) \\
*'I see the dog' IncorrectHET \\
Neuter & $\begin{array}{l}\text { Ik zie het schaap (12) } \\
\text { 'I see the sheep' CorrectHET }\end{array}$ & “' * zie de schaap (12) \\
& *I see the sheep' IncorrectDE \\
\hline
\end{tabular}

3.2 Experimental design

An experimental design is set up to "tap the knowledge" in monolingual and bilingual children with respect to their gender system of definite determiners. Spoken items are paired with an identical image on the screen of a laptop monitor The subjects have to decide as soon as possible whether the spoken item is correct or incorrect in Dutch.

They responded by pressing buttons on a button box. One button had a happy smiley face that the subjects pressed to indicate they believed the sentence to be correct Dutch, and another button had a sad smiley face that the subjects pressed if they believed the sentence to be incorrect Dutch.

The experiment is preceded by a practice sequence containing 4 spoken examples. Each subject receives this warm-up trial. After the practice session, two lists each with 24 spoken experimental items, are offered (see Table 4$).{ }^{5}$ Every experimental item consists of correct (CorrectDE: $I k$ zie de hond 'I see the dog and CorrectHET: $I k$ zie het schaap 'I see the sheep') and incorrect presentation (IncorrectHET: *Ik zie het hond '* see the dog'; and IncorrectDE: * Ik zie de schaap '*I see the sheep'). In other words, each presented noun is paired with a correct and an incorrect definite determiner. Moreover, a distinction is made between 12 common and 12 neuter nouns. ${ }^{6}$ All de- and het-words are unambiguous.

In addition to the 4 examples and 24 experimental items, 4 spoken filler items are used. The fillers consisted of a correct (e.g. Ik zie vandaag een kuiken, I see today a chicken') and an incorrect (e.g. ${ }^{*}$ Vandaag ik zie een kuiken, ${ }^{*}$ Today I see a chicken') word order.

5. The items resembled the ones used in the production study by Cornips et al. (2006).

6. Each gender type was also divided in 8 inanimate and 4 animate nouns. However, this article will not consider the role of animacy. 
3.3 Data analysis

This experiment yielded accuracy rates and response times as dependent variables. Language Group (monolingual versus bilingual children) was a between-subjects group factor. Presentation (correct versus incorrect items) was a within-language group factor. As illustrated in Table 4, in correct presentations (i.e. CorrectDE and CorrectHET), the determiner corresponds correctly to the gender of the noun, whereas in incorrect presentations (i.e. IncorrectDE and IncorrectHET), the determiner doesn't match with the gender of the noun. The factor Gender of noun (common de versus neuter het) varied between items.

First, accuracy rates were analysed using a MANOVA to examine differences between the monolinguals and bilinguals on the different item categories, i.e. CorrectDE; CorrectHET; IncorrectDE; and IncorrectHET (see \$4.2). Secondly, a Repeated Measures analysis was conducted to study response time differences between the Language Groups on the different item categories (see $\$ 4.4$, Baarda \& de Goede 1999; de Vocht 2005)

It is important to keep in mind that we cannot be sure what we are measuring when considering accuracy rates for the presented correct items. Hence, it might be the case that what we take as correct knowledge about the gender specification is due to a yes-bias, that is to say, the children prefer to press the 'smiley'- or 'yes-button' rather than the 'sad'- or 'no-button'. A potential 'yes'-bias can only be excluded if the same children also reveal high scores on the 'no'-button when confronted with incorrect items. Therefore, we will first compare the 'yes' with the 'no' scores.

\subsection{Predictions}

\section{We put forward the following predictions:}

Monolinguals. Since previous studies have found that older monolingual children show target-like behaviour in their use of $d e$ and het in speech, this suggests that they have good knowledge of the abstract underlying gender features. It is predicted that in the present decision task they will be highly accurate in accepting grammatical items and rejecting ungrammatical items, and it is also predicted that their response times to the ungrammatical items will be slower than to the grammatical items.

Bilinguals: (1) If bilingual children have a mapping problem, but not a representational problem, they will not differ from monolingual peers in their response patterns in the decision task. (2) If the bilingual children misrepresent the gender specification in the sense that they have de compatible both with common and neuter nouns, we expect them to show a lower rejection rate for incorrectDE than incorrectHET in the accuracy data. (3) If, however, they al together fail to represent abstract knowledge of gender features, we expect them to randomly accept/reject incorrectDE and incorrectHET.

Let us now turn to the results.

\section{4. $\quad$ Results}

First, an analysis of variance (ANOVA) is conducted to examine whether there are significant differences between the language groups. Secondly, a multiple analysis of variance (MANOVA) examines differences between the item categories. Finally, a T-test will check possible differences between the language groups. Several ANOVAs will be conducted to check the MANOVA (Baarda \& de Goede 1999; de Vocht 2005).

\subsection{Item-analysis}

To start with, all $d e$ - and het-items are controlled for reliability. The Cronbach's alpha with a value of 80 or higher in Table 5 shows that the test items were very reliable. ${ }^{7}$

Table 5. Reliability analysis

\begin{tabular}{ll}
\hline Items & Cronbach's alpha \\
\hline CorrectDE & .86 \\
IncorrectDE & .84 \\
CorrectHET & .83 \\
IncorrectHET & .80 \\
\hline
\end{tabular}

7. One of the reviewers recommended us to determine Cronbach's alpha on the separate language groups, because the bilinguals might be treating some terms aberrantly, while the monolinguals are not. This analysis showed that the bilinguals (alpha $=.53$ ) indeed performed less consistent on the accuracy items than the monolinguals (alpha $=.85$ ). However a subjectan cor in le could bave had an influence on the results. Clearly more research is necessary. 
Table 6. Accuracy rates (\% correct) for CorrectDE and CorrectHET

\begin{tabular}{lll}
\hline Language groups & \multicolumn{2}{c}{ Presentation } \\
& CorrectDE (de hond) & CorrectHET (het schaap) \\
\hline Monolinguals & 92 & 93 \\
Bilinguals & 70 & 80 \\
\hline
\end{tabular}

Table 7. Accuracy rates (\% correct) for IncorrectHET and IncorrectDE

\begin{tabular}{lll}
\hline Language groups & \multicolumn{2}{c}{ Presentation } \\
& IncorrectHET (*het hond) & IncorrectDE (*de schaap) \\
\hline Monolinguals & 91 & 88 \\
Bilinguals & 40 & 42 \\
\hline
\end{tabular}

4.2 Accuracy data: (in)correctDE and (in)correctHET

The results regarding the accuracy rates for the presented correct items are presented in Table 6. The monolinguals show higher accuracy rates than the bilinguals both on CorrectDE (92\% versus 70\%, respectively) and CorrectHET (93\% versus $80 \%$, respectively). This difference appears to be significant (CorrectDE: $p<.001$ and CorrectHET $p=.005$ ).

It is important to keep in mind that we cannot be sure what we are measuring in Table 6 when we consider accuracy rates for the presented correct items only. Hence, it might be the case that high rates are due to a preference of the children to press the 'smiley'- or 'yes'-button more than the 'sad'- or 'no'-button. A potential 'yes'-bias can only be excluded when the same children also press the 'no'-button. Let us therefore turn to the accuracy rates for the presented incorrect items.

Table 7 shows the accuracy rates for the presented incorrect items. Again, there is a difference between the monolingual and bilingual children in the accuracy rates for IncorrectHET (91\% versus $40 \%$ ) and IncorrectDE ( $88 \%$ versus $42 \%$ ). Thi difference is also significant $(p<.001)$ for both incorrect presentations.

Let us turn now to the consequences of these results for our hypotheses.

4.3 What about the accuracy rates hypotheses?

As mentioned above, the monolinguals show high accuracy rates on both the acceptance of the correct items and the rejection of the incorrect items. This means

8. It must be noted that the bilinguals showed more individual variation than the monolinguals in their performance. However, this is a well known phenomenon from the literature. they show consistent behavior. From this, it is safe to conclude that the monolinguals possess good knowledge of the Dutch gender specification.

In contrast, the bilinguals show a much higher accuracy rate on the acceptance of the presented correct items ('yes'-button; 70\% correctDE and $80 \%$ correctHET) than on the rejection of the incorrect items ('no'-button; $40 \%$ incorrectHET and $42 \%$ incorrectDE). One might think that the rather high accuracy rates for the correct items in Table 6 are due to a 'yes'-bias as a task-effect. However, as pointed out by an anonymous reviewer, such a task-effect should also affect the monolinguals and this is clearly not the case.

What does this mean for our hypotheses? First, the results do not support the mapping hypothesis, in the sense that it is certainly not the case that the bilingual children possess the right knowledge of gender specification in Dutch. The accuracy rates for the incorrect items are far too low ( $40 \%$ incorrectHET and $42 \%$ incorrectDE). Second, the results do not support the misrepresentation hypothesis either since the bilingual children do not show a lower rejection rate for incorrectDE than for incorrectHET. On the contrary, the random acceptance/rejection rates of incorrectDE and incorrectHET presents evidence in favour of the third hypothesis, indicating failure of gender specification. The results suggest that the bilingual children fail to represent abstract gender appropriately and therefore assume that $d e$ and het are in free variation in a decision task. But, they are clearly not in free variation for the monolingual children (and they are also not in free variation for the bilinguals in production, where de seems to be the default).

\subsection{Frequency-effect}

An interesting question that arises is whether the frequency of the test item c.q. noun plays a role in its correct assignment and rejection according to grammatical gender. The 24 singular nouns presented as test items can roughly be divided into three categories according to their frequency in the Spoken Dutch Corpus e.g. nouns that are:

i. very frequent - more than thousand tokens,

ii. frequent - more than 100 tokens and

iii. infrequent - less than 100 tokens (see Table 8 below). ${ }^{9}$

9. The Spoken Dutch Corpus is a database of contemporary standard Dutch as spoken by 9. The ad in Tetherlands and Flanders. The total number of words available is nearly 9 millio 800 hours of spech) Some 3.3 million words werecoll in The Netherlands. The corpus comprises a large number of samples of (recorded) spoken text (see http://lands.let.kun.nl/cgn/ehome.htm). 
Table 8. The frequency of the test items according to the Spoken Dutch Corpus

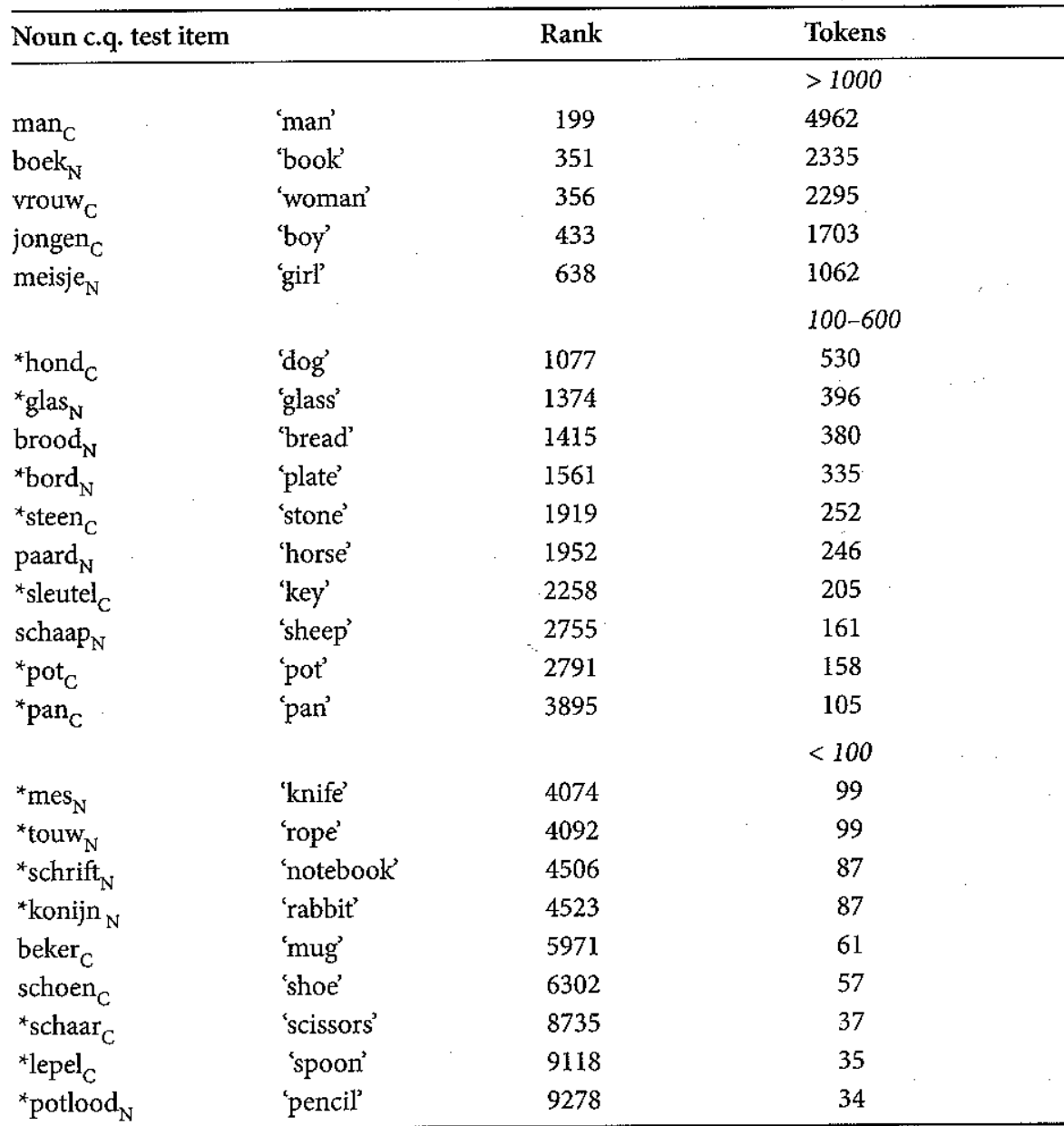

* inconsistent behavior

An item-analysis shows that overall the children give a correct response on the correct items (range: $73.8 \%-90.5 \%)$ whereas this is significant $(p<.000)$ less so on the incorrect items (range: $50 \%-78.6 \%$ ). Further, the most frequent nouns $(>1000)$ reveal a consistent pattern in the sense that by and large the children accept as much the correct items as they reject the incorrect ones (for example de man 'the man' and *het man have an acceptance and rejection percentage of 85,7 and 78,6 , respectively). However, 7 out of 10 nouns falling in a token range between 100 and 600 show a less consistent pattern and 7 out of 9 nouns belonging to the category infrequent nouns (less than 100) even show a very inconsistent pattern (for example, $88.1 \%$ of the children accepts the correct item het paard 'horse but only $26.2 \%$ rejects the incorrect de paard). The linkage between (in)frequency of the tokens and (in)consistent patterns reveal a frequency effect signalling that the bilingual acquisition of grammatical gender in Dutch is sensitive to input factors. ${ }^{10}$

\section{Response time data: (in)correctDE and (in)correctHET}

With respect to the response time data, if the bilingual children assume that $d e$ is compatible both with common and neuter nouns (misrepresentation), we expect that it will take more time to reject IncorrectDE $\left({ }^{*}\right.$ de schaap $\left._{N}\right)$ than IncorrectHET $\left({ }^{*}\right.$ het hond $\mathrm{d}_{\mathrm{C}}$ ). In contrast, if they have good knowledge of grammatical gender in Dutch (mapping problem), we don't expect to find any substantial differences in response time data regarding rejecting incorrect $d e$ and incorrect het although the rejection of incorrect items will take some more time than accepting the correct presentations

Table 9 presents the response time for the presented CorrectDE and CorrectHET items. There are no major differences between the monolinguals and bilinguals, that is, they both need the same amount of time to push the 'yes'-button. Moreover, the monolingual and bilingual children do not differ in response times regarding $d e$ - and het-items.

Let us turn now to Table 10 showing response times for the presented IncorrectHET and IncorrectDE items.

Table 9. Response times (in ms) for CorrectDE and CorrectHET

\begin{tabular}{lll}
\hline Language groups & \multicolumn{2}{c}{ Presentation } \\
& CorrectDE (de hond) & CorrectHET (het schaap) \\
\hline Monolinguals & 1495 & 1554 \\
Bilinguals & 1584 & 1490 \\
\hline \multirow{3}{*}{ Table 10. Response times (in ms) for IncorrectHET and IncorrectDE } \\
\hline Language groups & \multicolumn{2}{c}{ Presentation } \\
& IncorrectHET (*het hond) & IncorrectDE (*de schaap) \\
\hline Monolinguals & 1585 & 1577 \\
Bilinguals & 1765 & 1640 \\
\hline
\end{tabular}

o. All the children who display inconsistent behaviour are bilingual, none of them are monolingual. 
We see that for both the monolingual and the bilingual children, it takes more time to reject the presented incorrect items (Table 10) than accepting the correct ones (Table 9) $(p<.001)$

\subsection{What about the response times hypotheses?}

The response times of both the monolinguals and the bilinguals confirm gen eral psycholinguistic findings that the rejection of presented incorrect items takes longer than the acceptance of the presented correct items, although the monolinguals perform faster than the bilinguals.

Furthermore, the results in Table 10 show that it did not take the bilinguals longer to reject incorrectDE than incorrectHET, consequently there is no evidence in favor of the misrepresentation hypothesis. Indeed, it took them a bit longer to reject incorrectHET than to reject incorrectDE, suggesting at least some knowledge about grammatical gender, contrary to what we concluded on the basis of the accuracy results. Clearly, further research is necessary here.

\section{Conclusion}

Our starting point was that previous research revealed that monolingual children between 11 and 13 years old show a target-like production with respect to gender assignment of definite determiners whereas this is not the case for bilingual children of the same age who massively overgeneralize $d e$.

In order to further investigate how to interpret this overgeneralization of de in the production data of bilingual children between 11 and 13 years old, we designed an experimental decision taks, "tapping the knowledge" of both monolingual and bilingual children. As for what we expected to find, we formulated three hypotheses.

First, if bilingual children just have a mapping problem they will not differ from monolingual peers in their response patterns in the decision task.

Our results show that this is not the case: they reveal a significantly lower percentage of incorrect items than the monolinguals i.e. only $40 \%$ (vs. $90 \%$ for the monolinguals)

Second, if the bilingual children misrepresent the gender specification in the sense that they assume that $d e$ is compatible with both common and neuter nouns, we expect them to show a lower rejection rate for incorrectDE than incorrectHET.
Our results show that this is not the case either: the accuracy rates for incorrectHET and incorrectDE are $40 \%$ and $42 \%$, respectively.

This, in fact, might support the third hypothesis, namely that they fail to represent abstract gender in their grammar and that $d e$ and het are in free variation in the decision task.

Nevertheless, with respect to the bilingual children, we attested a difference between the original production data which show a massive overgeneralization of $d e$ and the accuracy data which do not show such a difference between de and het. We saw, however, that the response time data did suggest some awareness of grammatical gender in the bilingual children, since they needed more time to reject incorrectHET than to reject incorrectDE. If these observations are correct and indicate that the bilingual children have some awareness of de and het being gender markers, the overgeneralization in the production data is possibly not a grammatical phenomenon, but more likely a speech production strategy.

We like to speculate that the present results in combination with the production data might indicate that, initially, young children have no gender-specification in their grammar. ${ }^{11}$ They only use the feature specification [definite] for which they choose $d e$ as the 'default' value, possibly due to its overwhelming presence in the input. So, they first have no idea that gender plays a role. At a certain moment, ${ }^{12}$ they become aware of [gender] as an abstract grammatical specification. It might be the case that at first the feature-specification of gender remains underspecified. In other words, then they are aware that $d e$ and het are related to grammatical gender, but they don't know the right gender specification (yet). In that stage, they not only overgeneralize de to neuter nouns, but they also sometimes overgeneralize het to common nouns (see Table 3). Monolingual children overcome this stage and acquire good knowledge of gender specification, whereas bilingual children possibly remain in this stage, i.e. they might fossilize. Further research will have to show what makes it so difficult for bilingual children to leave this stage and become targetlike in the acquisition of grammatical gender. Specially since such a "fossilization effect" has not been found for the acquisition pattern of other grammatical phenomena in these children, such as word order (see Hulk \& Cornips 2006). Several possibilities have to be considered, involving not only psycholinguistic and grammatical factors, but also sociolinguistic factors.

11. An anonymous reviewer pointed out to us that, given the literature, this implies that the acquisition of grammatical gender would differ from the acquisition of grammatical number.

12. See Cornips, Hulk \& Brouwer (2007) for a suggestion about a possible trigger. 


\section{Acknowledgements}

We are grateful to the editors of this volume, and to two anonymous reviewers for insightful comments and suggestions on earlier versions of this paper. This work has been done at Utrecht University in fulfillment of an internship.

\section{References}

Baarda, D. B. Goede, M. P. M. de 1999. Basisboek Statistiek met SPSS voorWindows. Groningen: Wolters-Noordhoff.

Blom, E., Polisenská, D. \& Weerman, F. 2006. Effects of age in the acquisition of gender: A three-way distinction between child L1, child L2 and adult L2 acquisition. Talk presented at Amsterdam Gender Colloquium given on September 16th, Vrije Universiteit, Amsterdam.

Bol, G. W. \& Kuiken, F. 1988. Grammaticale Analyse van Taalontwikkelingsstoornissen. PhD dissertation, University of Amsterdam.

Cornips, L., Hulk, A. \& Brouwer, R. 2007. Diminutives and gender awareness in bilingual acquisition. Talk presented at ISB6 given on June 2 nd, Hamburg.

Cornips, L., Hoek, M. van der \& Verwer, R. 2006. The acquisition of grammatical gender in bilingual child acquisition of Dutch (by older Moroccan and Turkish children). The definite determiner, attributive adjective and relative pronoun. In Linguistics-in-The Netherlands, B. Los \& J. van de Weijer (eds), 40-51.

De Houwer, A. \& Gillis, S. 1998. The Acquisition of Dutch. Amsterdam: John Benjamins.

Hulk, A. \& Cornips, L. 2006a. Neuter gender and interface vulnerability in child L2/2L1 Dutch. In Paths of Development in L1 and L2 acquisition: In Honor of Bonnie D. Schwartz, S. Unsworth et al. (eds), 107-134. Amsterdam: John Benjamins.

Hulk, A. \& Cornips, L. 2006b. Between 2L1 - and child L2 acquisition: An experimental study of bilingual Dutch. In Interfaces in Multilingualism: Acquisition and representation. Hamburg Studies on Multilingualism, Vol. 4, C. Lleo (ed.), 115-137, Amsterdam: John Benjamins. Spoken Dutch Corpus, see http://lands.let.kun.nl/cgn/ehome.htm.

Velde, M. van der 2002. Determiner acquisition in Dutch and French. Talk presented at TIN dag (Linguistics in the Netherlands) given on January 26th, Utrecht University.

Velde, M. van der 2004. L'acquisition des determinants en L1: Une etude comparative entre le Francais et le Néerlandais. Acquisition et interaction en langue etrangere 21: 9-46.

Vocht A de 2005. Basishandboek SPSS 11 voor Windows. Utrecht: Bijleveld Pres.

\section{Comparing child and adult $\mathrm{L} 2$ acquisition of the Greek DP}

Effects of age and construction*

\author{
Vicky Chondrogianni \\ University of Cambridge
}

The present paper investigates the acquisition of the definite article and third person direct object clitics by Turkish-speaking L2 learners of Modern Greek (henceforth Greek). Oral production data was elicited using identical methods from child and adult L2 learners who belong to similar proficiency levels. The results show an asymmetrical pattern in the acquisition of the two D-elements, with the acquisition of the clitic being severely delayed. This can partly be explained by theories that assume transfer in L2 acquisition, but pose problems to the ones suggesting lack of attainment due to feature interpretability. The paper discusses additional factors that may account for the acquisition pattern, such as the linguistic structure to be acquired coupled with notions of the age of exposure and the quantity of input received.

\section{Introduction}

A much debated topic in the second language acquisition (L2A) literature is whether and to what extent adult L2A is guided by the same underlying mechanisms as L1 acquisition (L1A), and more particularly, by Universal Grammar (UG). Unlike children who ultimately and uniformly reach nativelike competence in their L1, for (adult) L2 learners successful acquisition of L2 properties is not guaranteed. 'Defective' acquisition has been attributed to the age of exposure and to the

* I would like to thank Belma Haznedar for giving me the opportunity to participate in this volume. I also gratefully acknowledge the comments of the two anonymous reviewers. I would

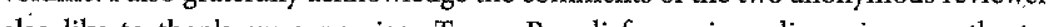
also like to thank my supervisor Teresa Pardh for vanious discussions on the topic. Great thanks go to Marios Mavrogiorgos for exchange of ideas. All errors of course remain my own. This research was funded by the Economic and Social Research Council and the University of Cambridge, UK. 